\title{
Effect of His Bundle Pacing on Right Bundle Branch Block Located Distal to Site of Pacing.
}

\author{
Rehan Mahmud $^{1}$, Shakeel Jamal ${ }^{1}$, and Brenda Harris ${ }^{1}$ \\ ${ }^{1}$ McLaren Bay Region
}

September 16, 2020

\begin{abstract}
Aims: it is generally accepted that bundle branch block (BBB) may be corrected simply by pacing (P) the His bundle (HB) distal to site of block. This hypothesis, based on observations with percutaneous catheters, assumes that conduction block is in proximal HB. However, these postulations have not been systematically studied following active fixation of HB pacing lead. We analyzed role of pacing voltage and capture thresholds in selective (S) and non-selective (NS) HBP in patients with right (R) BBB. Methods: In thirty-nine patients with RBBB, 4 showed S-HBP, 18 showed NS-HBP, and 17 showed NS-HBP at $>2.4 ? 0.8$ $\mathrm{V}$ and S-HBP at lower voltage (NS-S HBP group). Results 1. During S-HBP there was no correction of RBBB. 2. NS-HBP either completely or partially corrected RBBB along with with a decrease in QRS activation time (91?9ms from 98 ?6ms). 3. NS-HBP group with capture threshold of $1.3 ? 0.5 \mathrm{~V}$ completely resolved RBBB in 9/14 vs 3/11 patients in NS-S HBP group with higher capture threshold of 2.4?0.8V. 4. During NS-HBP higher voltage caused complete resolution of RBBB in 22/39 patients vs 10/39 at lower voltage. Conclusions: 1.) Lack of correction with S-HBP suggests that RBBB was distal to site of HBP and yet was corrected with NS-HBP. 2.) Voltage dependent properties in NS-HBP suggests that conduction via a specialized parallel pathway maintains normal ventricular activation time. 3.) Correction of RBBB in all patients with NS-HBP, suggests that conduction block was either bypassed or right ventricular free wall pre-excited by conduction via a parallel pathway.
\end{abstract}

Title Page

Effect of His Bundle Pacing on Right Bundle Branch Block Located Distal to Site of Pacing. Rehan Mahmud MD,

McLaren Bay Region,

1900 Columbus Ave, Bay City MI 48708, USA

Corresponding author : drmahmud@msn.com

Shakeel Jamal, MD

McLaren Bay Region,

1900 Columbus Ave, Bay City MI 48708 USA

jamal1sm@cmich.edu

Brenda Harris RN

McLaren Bay Region,

1900 Columbus Ave, Bay City MI 48708, USA

drmahmud@msn.com 


\section{Abstract}

Aims: it is generally accepted that bundle branch block (BBB) may be corrected simply by pacing $(\mathrm{P})$ the His bundle (HB) distal to site of block. This hypothesis, based on observations with percutaneous catheters, assumes that conduction block is in proximal HB. However, these postulations have not been systematically studied following active fixation of HB pacing lead.

We analyzed role of pacing voltage and capture thresholds in selective (S) and non-selective (NS) HBP in patients with right $(\mathrm{R}) \mathrm{BBB}$.

Methods: In thirty-nine patients with RBBB, 4 showed S-HBP, 18 showed NS-HBP, and 17 showed NS-HBP at $>2.4 \pm 0.8 \mathrm{~V}$ and S-HBP at lower voltage (NS-S HBP group).

Results

1. During S-HBP there was no resolution of RBBB.

2. NS-HBP completely or partially resolved RBBB along with a decrease in QRS activation time $(91 \pm 9 \mathrm{~ms}$ from $98 \pm 6 \mathrm{~ms})$.

3. NS-HBP group with capture threshold of $1.3 \pm 0.5 \mathrm{~V}$ completely resolved RBBB in $9 / 14$ vs $3 / 11$ patients in NS-S HBP group with higher capture threshold of $2.4 \pm 0.8 \mathrm{~V}$.

4. During NS-HBP higher voltage caused complete resolution of RBBB in $22 / 39$ patients vs 10/39 at lower voltage.

Conclusions:

1. Lack of correction with S-HBP suggests that RBBB was distal to site of HBP and yet was corrected with NS-HBP.

2. Voltage dependent properties in NS-HBP suggests that conduction via a specialized parallel pathway maintains normal ventricular activation time.

3. Correction of RBBB in all patients with NS-HBP, suggests that conduction block was either bypassed or right ventricular free wall pre-excited by conduction via a parallel pathway.

Introduction

In His bundle pacing (S-HBP) it is generally assumed that conduction block is in proximal His bundle and pacing distal to site of block results in normal QRS complex (1-4).

However, the site of His bundle pacing is predicated on recording a His bundle electrogram and achieving acceptable pacing threshold and moving the lead distally along the His bundle is not always an option. It would seem plausible therefore that correction of HPS conduction block may be due to mechanisms other than serendipitously pacing from a site distal to site of block.

Right bundle branch block (RBBB) presents a unique opportunity to study the mechanisms involved as acute RBBB seen following lead fixation may well be in close proximity yet 'distal' to pacing lead tip location, whereas chronic RBBB may be in either proximal or distal.

Furthermore, in RBBB the conduction delay primarily affects terminal QRS vectors and is less likely to be directly affected by NS-HBP which alters the initial portion of QRS.

Methods

Definitions: Selective (S) and non-Selective (NS) His bundle pacing (HBP) have been defined before (2). In general S-HBP had an isoelectric stimulus to QRS interval and NS-HBP had a delta wave like abnormality following the stimulus.

Acute $R B B B$ was defined as right bundle branch block which occurred during active fixation of the lead. A pre-existing RBBB was labeled as chronic $R B B B$. 
Patient population: An ethical informed consent approved by the relevant hospital institution was obtained from each patient along with the patient's consent to publish de-identified data. All analysis was done on stored data routinely obtained during pacemaker implant.

An audit of past 300 consecutive patients who had undergone His bundle pacing revealed 27 patients with chronic RBBB and 12 patients who developed acute RBBB which persisted throughout the implant procedure. All patients had undergone a standardized pacing threshold protocol. Using $1.0 \mathrm{~ms}$ bipolar pulse width, starting at $5 \mathrm{~V}$ with stepwise decrements to $1 \mathrm{~V}$. A 12 lead electrocardiogram (ECG) at each pacing voltage was labeled and stored for review.

The patients were further divided into 3 groups based on whether observable conduction was occurring via a) His bundle, a parallel pathway or both:

1. Both NS-HBP and S-HBP was observed (NS-S HBP group)

2. Only NS-HBP was observed (NS-HBP group):

3. Only S-HBP was observed (S-HBP group):

We did not identify any patient with S-HBP at high voltage with transition to NS-HBP at lower voltage.

Method of implantation of the Medtronic 3830 HBP lead has been previously described (5).

Site of implantation: In brief, we required that post lead fixation $\mathrm{H}-\mathrm{V}$ interval was $>35 \mathrm{msec}$ and that the stimulus to peak ventricular activation time at $5 \mathrm{~V}$ was not $>10 \mathrm{msec}$ longer than baseline His- peak ventricular activation time, otherwise the lead was relocated.

\section{Measurements:}

Following active fixation of His bundle pacing lead a baseline $\mathrm{H}-\mathrm{V}$ interval plus ventricular activation time (VAT) in Lead 1 was measured and designated as baseline HVAT interval (Fig.1)

In addition, the following intervals were measured during paced beats at each

$1-\mathrm{V}$ decrement.

1. The interval from His bundle pacing artifact $(\mathrm{H})$ to the peak deflection of the QRS in Lead 1, i.e., His $(\mathrm{H})$ to peak ventricular activation time (VAT), designated as HVAT of paced beat) (Fig. 1).

2. Stimulus to QRS (Stim-q) interval in S-HBP (Fig. 1).

3. Lead I voltage (Fig. 1).

4. QRS axis in frontal plane was measured using an online ECG analysis tool.

5. V lead transition in chest leads.

We used a Cardiolab (GE Healthcare) signal acquisition and recording system. The 12 lead ECG filter setting was $0-100 \mathrm{~Hz}$. The His bundle electrogram

Study data including QRS morphology was analyzed offline on a review monitor. Activation times were measured using an on-screen multi-leg caliper.

Statistics: A paired t test was applied to the data, using an online statistics calculator (GraphPad). Mean difference at a $\mathrm{p}$ value $<0.05$ was considered significant.

Results

Threshold testing resulted in non-selective (NS) and selective (S) His bundle pacing (HBP as follows.

1. NS-S HBP group: Both NS and S HBP was observed in 17 patients (11 chronic and 6 acute RBBB).

2. NS-HBP group): Only non-selective HBP was observed in 18 patients (14 chronic, 4 acute)

3. S-HBP group: Only selective HBP was see in 4 patients (2 chronic, 2 acute). 
The changes in baseline characteristics are shown in Table 1. The His Purkinje system-peak ventricular activation time (HVAT), showed no change with S-HBP however slight but significant decrease was seen with NS-HBP. Whereas the

S-HBP complex was nearly identical to baseline, the NS-HBP complex showed characteristic changes which included a significant increase in L1 voltage (all patients) as well as a significant

counterclockwise rotation observed as rightward transition in chest V leads (Table 1)

However, there was no change in frontal QRS axis with NS-HBP which remained in normal range in all patients.

Table 1. Changes in baseline characteristics in NS-HBP and S-HBP

\begin{tabular}{llll}
\hline $\mathbf{n}=\mathbf{1 7}$ & Baseline & Non selective-HBP @ & \\
\hline HVAT (ms) & $\mathbf{5 V}$ & Selective HBP \\
$\begin{array}{l}\text { LEAD 1 VOLTAGE } \\
(\mathrm{mV})\end{array}$ & $0.6 \pm 0.3$ & $91 \pm 9 \mathrm{P}=0.04$ & $90 \pm 7 \mathrm{P}=\mathrm{ns}$ \\
$\begin{array}{l}\text { FRONTAL AXIS } \\
\text { degrees) }\end{array}$ & $38 \pm 23^{\circ}$ & $1.02 \pm 0.3 \mathrm{P}=0.004$ & $0.5 \pm 0.3$ \\
$\begin{array}{l}\text { V LEAD } \\
\text { TRANSITION }\end{array}$ & $\mathrm{V} 4$ & $34 \pm 30^{\circ}$ & $36 \pm 28^{\circ}$ \\
\hline
\end{tabular}

Analysis by patient groups

NS-S Group:

Acute $\mathrm{RBBB}=6$, Chronic $\mathrm{RBBB}=11$.

These patients transitioned from NS-HBP to S-HBP at a mean pacing voltage of $2.4 \pm 0.8 \mathrm{~V}$ (capture threshold of NS-HBP). The capture threshold of S-HBP was $1.0 \pm 0.7 \mathrm{~V}$.

The baseline $\mathrm{H}-\mathrm{V}$ interval was $53 \pm 8 \mathrm{~ms}$. Following transition to S-HBP the stim-q interval was $57 \pm 10 \mathrm{~ms}$.

In patients who developed acute RBBB with active fixation, the RBBB was completely reversed with NSHBP phase (from $5.0 \mathrm{~V}$ to $>2.4 \pm 0.8 \mathrm{~V}$ ), however upon transition to S-HBP phase complete RBBB recurred in all patients in this group (Fig. 1).

In patients with chronic RBBB, either partial (8/11) or complete correction of RBBB (3/11 patients) occurred but only during the NS-HBP phase at pacing voltage $>2.4 \pm 0.8 \mathrm{~V}$. Like acute RBBB, in all 11 patients with chronic RBBB recurred on transition to S-HBP phase (Figures 2,3).

Of the 9 patients with complete correction of RBBB, in 2 patients with acute RBBB and 1 patient with chronic RBBB as pacing voltage was lowered, a progressive increase in rightward delay was seen (Figs. 1,4). This voltage effect was seen only with NS-HBP and not during S-HBP where abrupt recurrence of complete RBBB was seen in all patients.

Figure1: Effect of NS-HBP and S-HBP in acute RBBB 

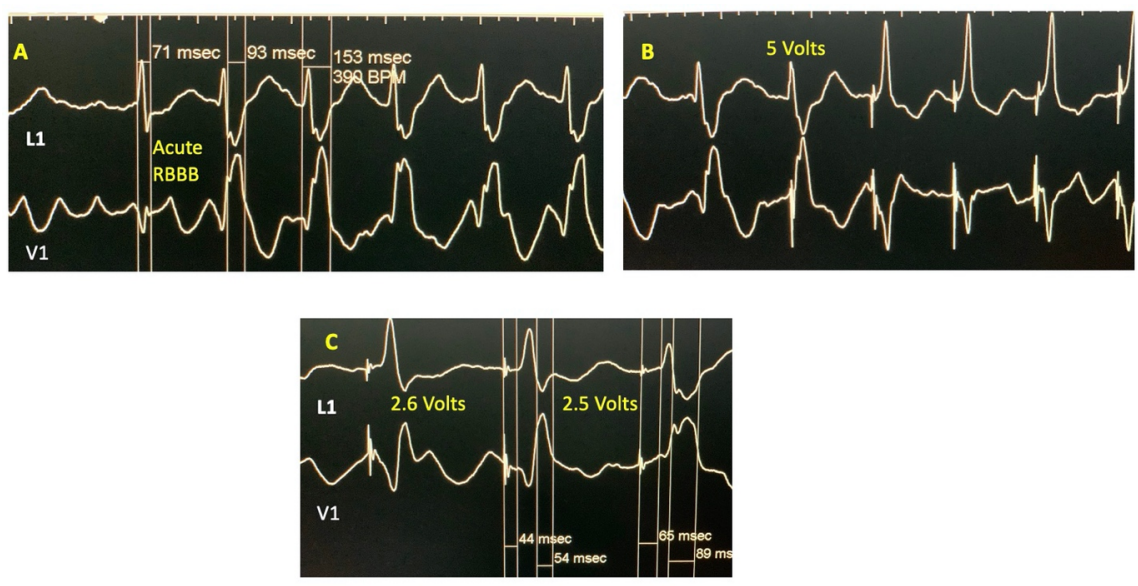

All figures show surface ECG recordings at $100 \mathrm{~mm} / \mathrm{sec}$

Panel $A$ shows the development of acute $R B B B$ during active fixation of the His bundle pacing lead, suggesting that pacing site was not be distal to site of block.

In panel $B$, complete resolution of $R B B B$ is seen with $5 \mathrm{~V}$.

In panel $C$, note development of progressively greater $R B B B$ (wider $S$ wave in $L 1$ is seen as pacing voltage is lowered until complete RBBB occurs upon transition to selective HBP (last beat) which shows that site of block is 'distal' to pacing site.

Figure 2: Partial resolution of RBBB with of NS-HBP, unchanged by pacing voltage. 

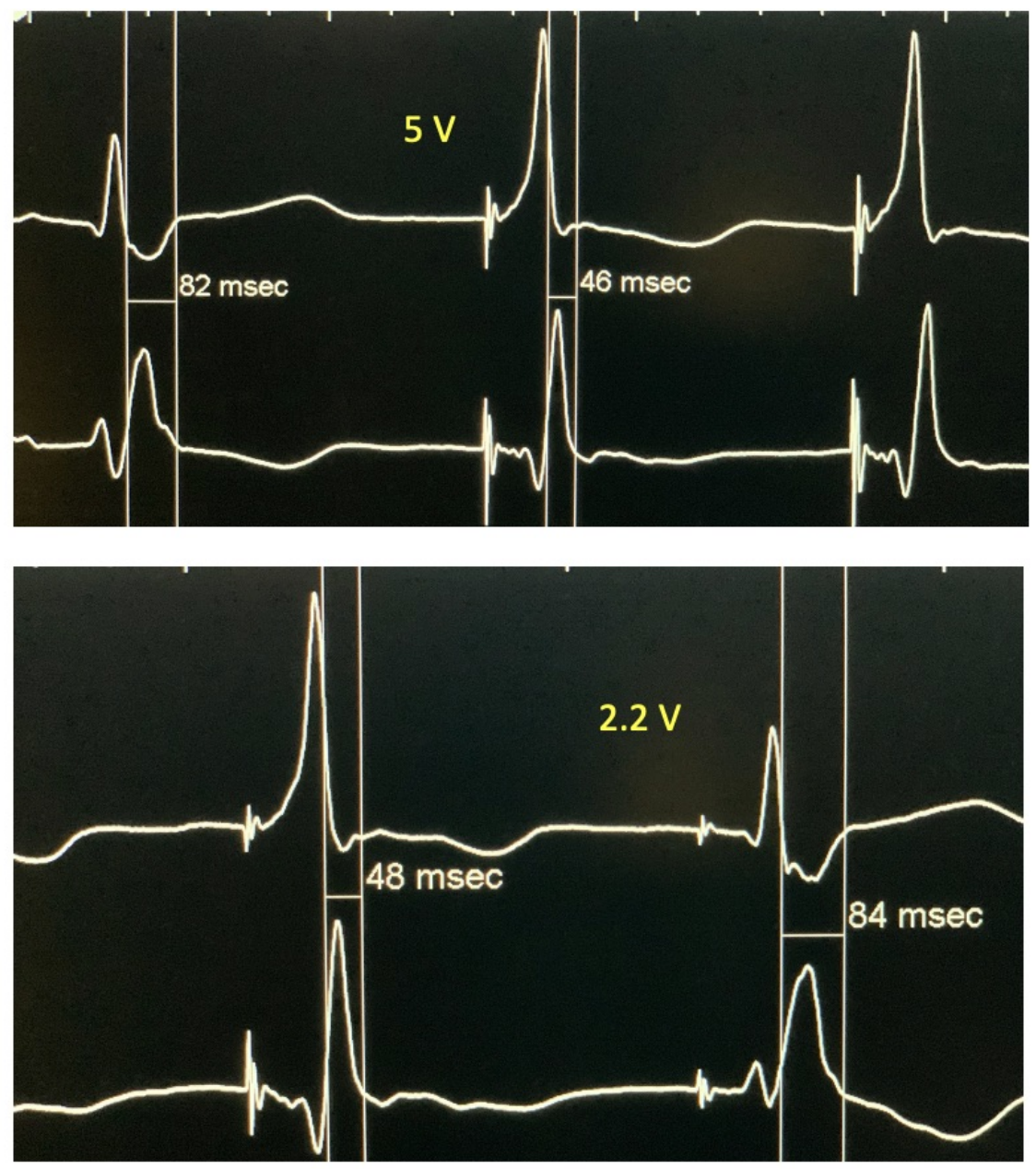

Upper panel: In a patient with chronic RBBB (see first sinus beat) pacing at 5 volts results in partial correction of RBBB with $L 1 S$ wave decreasing from $82 \mathrm{msec}$ to $46 \mathrm{msec}$.

Lower panel: There is no change L1S wave and V1 r', as pacing voltage is lowered (first beat). However, as transition to $S$-HBP occurs (second beat) there is abrupt recurrence of complete $R B B B$.

This figure shows that RBBB was only partially corrected with NS-HBP and not affected by decrease in pacing voltage in NS-HBP, The complete recurrence of RBBB upon transition to S-HBP documents that the site of $R B B B$ was distal to pacing site.

Figure 3: Complete correction of a distal site of chronic RBBB with NS-HBP but not with S-HBP 

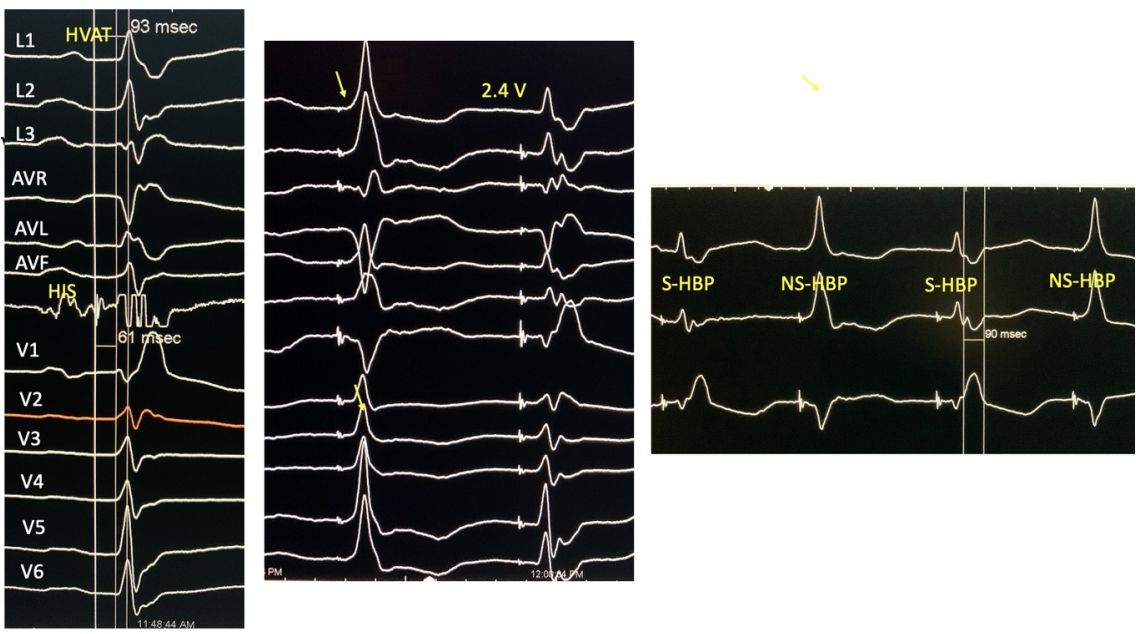

Left panel: Shows 12 lead ECG of a sinus beat with RBBB with a 'clipped' high gain His electrogram.

Middle panel: This patient showed complete resolution of $R B B B$ at $5 \mathrm{~V}$ and as capture threshold is reached at $2.4 \mathrm{~V}$ transition to $S$-HBP occurs with abrupt recurrence of complete $R B B B$.

Note the pre-excited beat maintains a normal frontal QRS axis, there is increase in L1 voltage and counterclockwise rotation in chest leads, from V5 (left panel, sinus beat) to V2 during NS-HBP (first beat middle panel). With $S-H B P\left(2^{\text {nd }}\right.$ beat, middle panel) counterclockwise rotation is no longer seen.

Right panel: This patient developed transient NS-HBP alternating with S-HBP with complete resolution and complete recurrence of $R B B B$ respectively.

This figure suggests that complete resolution of $R B B B$ occurs only when a parallel pathway causing the delta wave is activated.

Figure 4: Pacing voltage dependent correction of RBBB with NS-HBP

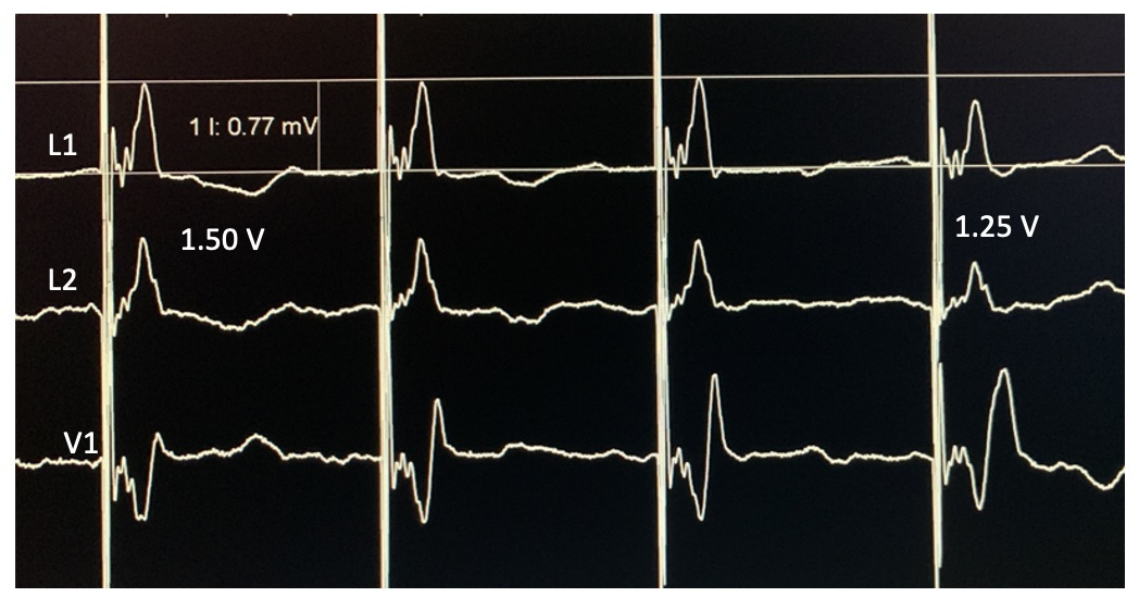

This figure shows progressive increase in rightward delay, best seen in Lead V1, as the pacing voltage is lowered from $1.5 \mathrm{~V}$. The fall in Lead 1 voltage (last complex) marks the transition from NS-HBP to S-HBP where abrupt development of complete RBBB is seen. This slide shows decreasing effect of parallel wavefront which resolves RBBB as the voltage is lowered. 


\section{NS-HBP Group:}

Acute $\mathrm{RBBB}=4$, Chronic $\mathrm{RBBB}=14$

These patients showed only NS-HBP. The baseline characteristics were similar to NS-S group however the capture threshold of the pre-excitation wave now occurred at lower pacing voltage of $1.3 \pm 0.5 \mathrm{~V}$ compared to $2.4 \pm 0.8 \mathrm{~V}$ in NS-S HBP group. The mean H-V interval was $52 \pm 5 \mathrm{~ms}$. The HVAT at baseline was $98 \pm 6 \mathrm{~ms}$ and decreased to $89 \pm 5 \mathrm{~ms}$ with $5 \mathrm{~V}$ pacing.

All 4 patients with acute RBBB showed complete resolution of RBBB at $5 \mathrm{~V}$ pacing 2 of the 4 showed partial recurrence of $\mathrm{RBBB}$ at lower voltage.

Of 14 patients with chronic RBBB, 9 had complete resolution at $5 \mathrm{~V}$ and 5 had incomplete resolution at both high and low voltage pacing. In 9 patients with complete resolution at 5V, 6 developed partial recurrence at lower voltage during NS-HBP.

Thus in 8 patients (2 acute and 6 chronic), RBBB showed a voltage effect where complete resolution was seen at $5 \mathrm{~V}$ however the only partial correction at lower voltage.

\section{S-HBP Group:}

\section{Acute $\mathrm{RBBB}=2$, Chronic $\mathrm{RBBB}=2$.}

Only 4 patients showed S-HBP at both high and low pacing voltages.

The mean H-V interval was $57 \pm 12 \mathrm{~ms}$, the baseline HVAT interval was $108 \pm 19$ and decreased to $97 \pm 15 \mathrm{~ms}$ with S-HBP.

Acute RBBB (2 patients): Only 1 patient with acute RBBB resolved completely with $5 \mathrm{~V}$ although NS-HBP could not be ruled out. Complete RBBB recurred at 3.5V (Fig.5).

Chronic RBBB (2 patients). Neither complete nor incomplete resolution of RBBB was seen at any voltage (Fig. 5).

Figure 5: Effect of 5V pacing on RBBB in S-HBP
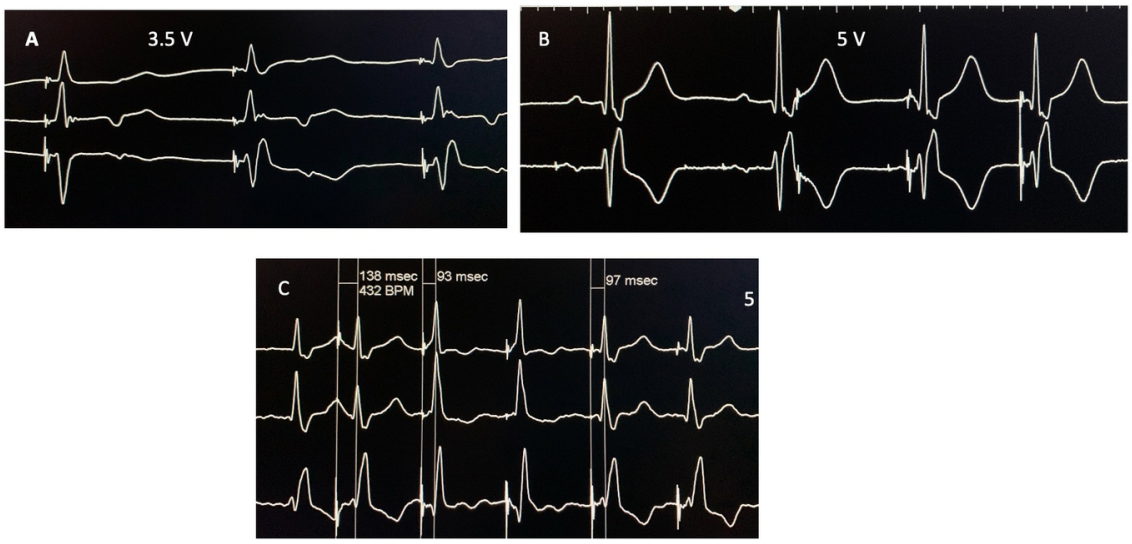

Panel A: Shows one patient with S-HBP where a transient but complete correction of acute RBBB appears to occur with S-HBP with recurrence as voltage was lowered to $3.5 \mathrm{~V}$. However, if corrected and uncorrected beats are compared (first and second beats) there are subtle differences (L1 voltage and initial $\mathrm{r}$ in V1) suggesting that septal activation may have been different in corrected beat and an alternate pathway may have been activated. 
Panel B: In remaining 3 patients with RBBB (one acute and 2 chronic) did not resolve with selective His bundle pacing at any pacing voltage.

Panel C: In one patient, chronic RBBB resolves partially, however with transient NS-HBP $\left(3^{\text {rd }}\right.$ and $4^{\text {th }}$ beats) but recurs abruptly as selective HBP occurs with voltage still at $5 \mathrm{~V}$.

In patients in S-HBP group, the site of block was distal to pacing lead and was not resolved with S-HBP.

Note HVAT intervals in NS-HBP and S-HBP of 93msec and 97 msec respectively.

Discussion

The observation that both acute and chronic right bundle branch block (RBBB) remained uncorrected with selective His bundle pacing (S-HBP) clearly suggests that site of RBBB was distal to pacing lead tip (Fig. 1-4). Intra-operative study of conduction times and velocity would indicate that the mean $\mathrm{H}-\mathrm{V}$ intervals of $53 \pm 11 \mathrm{~ms}$ obtained in our study would place the lead tip in proximal His bundle; $2-3 \mathrm{~cm}$ from the bifurcation of His bundle into bundle branches and the ventricular septum (6). Even so, the RBBB was partially or completely corrected in all patients with non-selective (NS)-HBP where a characteristic 'delta' wave indicating that a parallel pathway, in addition to the His bundle, is also being activated (Fig. 1-4). From same pacing site, even in acute RBBB ostensibly located in close proximity to pacing lead, conduction block was rarely corrected with S-HBP.

Correction of RBBB appeared to correlate with pacing voltage, as higher pacing voltage $(5 \mathrm{~V})$ resulted in complete correction of RBBB in 22 patients while only 10 showed complete correction at lower voltage. The voltage effect including a unique pattern of progressive beat by beat correction of RBBB with higher voltage was seen only during NS-HBP . Complete recurrence of $R B B B$ was seen only with $S$-HBP never with NS-HBP (Fig. 1,4).

NS-HBP group showing lower capture threshold of the delta wave $(1.3 \pm 0.5 \mathrm{~V}$,$) had more patients with$ complete resolution of RBBB (9/14 patients) as compared to NS-S HBP group where the delta wave had higher capture threshold $(2.4 \pm 0.8 \mathrm{~V})$ and only $3 / 11$ patients had complete correction of RBBB.

Voltage related resolution of slow conduction has been observed in injured Purkinje fibers where higher voltage is required to generate a propagating impulse (7-10) and our observations during NS-HBP suggests that the parallel pathway may have properties similar to specialized conduction fibers. Increased pacing threshold is also predicted by virtual electrode polarization (VEP) theory and is felt to occur as a result of tissue damage $(11,12)$. Based on planar model of cardiac muscle, the VEP theory does not explain how higher voltage would result in improvement of conduction particularly in His Purkinje system. In our study, in acute RBBB in which injured His bundle fibers are in close proximity to pacing lead tip a clinically significant VEP effect was not readily apparent in S-HBP where conduction is exclusively via His bundle.

The explanation why the parallel pathway in NS-HBP demonstrates voltage dependent phenomenon, may lie in the work of Effimov group which shows evidence of parallel pathways in the His region (13). The His bundle and surrounding area shows a rich density of gap junction proteins ( $\mathrm{Cx} 43, \mathrm{Cx} 40$, and $\mathrm{Cx} 45)$ extending from right lower extension (RLE) of the AV node to the interatrial septum and the latter has more than twice the expression of CX-43 compared to the His bundle $(13,14)$. Thus, a molecular compartmentalization exists in the peri- Hisian region which connects directly to a specific Cx43-positive domain of the His bundle (13). Hucker et al. have suggested that as these molecular pathways are not encased in fibrous tissue, pacing the RLE would not only activate the His bundle but may require lower voltage than direct pacing of His bundle itself (13).

Thus, one may speculate that the pacing lead may disrupt and activate subendocardial tissues rich in gap junction proteins which may behave as a parallel specialized conduction pathway injured by the active fixation process.

Thus, If one assumes as our observations suggest, that conduction through normal pathway (His bundle) results in $R B B B$ and conduction through parallel pathway results in correction of $R B B B$ then robust conduction 
through the parallel pathway at higher voltage may completely resolve RBBB while at lower pacing voltage, slower conduction in the parallel pathway would allow conduction through His bundle to manifest the RBBB and progressive slowing in the parallel pathway would result in progressively greater RBBB (Fig.1,4).

Two possible mechanisms whereby interaction between the two wavefronts may resolve RBBB is depicted in Figure 6. In one possibility, early excitation of the RV free wall may decrease or abolish ECG evidence of RBBB. This mechanism is suggested by the counterclockwise rotation of the NS-HBP complex (Table 1, Fig. 3), a consistent feature of NS-HBP (15) it may result from an early rightward wavefront.

The work of Durrer et al $(16,17)$ and more recently of Almeida et al $(18,19)$ lays out the specialized conduction pathway to early RV free wall pre-excitation in NS-HBP. In normal QRS activation (Fig.6), following left to right septal activation, the wavefront proceeds to the septal border of the crista supraventricularis (CSV) and meets the RV free wall wavefront exiting from right bundle branch (16-19)

(Fig. 6, left upper panel). During NS-HBP early activation of septum would also result in activation proceeding anteriorly to the crista supraventricularis (CSV) which alone connects the septum to RV free wall (20). This early activation wavefront, upon finding the RV free wall unexcited because of RBBB, would proceed to pre-excite the RV free wall (fig. 6, right upper panel), thus decreasing the duration of the S wave in Lead 1 without actually resolving the conduction delay at the distal site. This mechanism also explains why allpatients, particularly those in whom conduction block may be located more distally in the right bundle branch, showed at least partial 'correction' of RBBB .

The right lower panel in figure 6 shows another mechanism where the parallel pathway bypasses a more proximally related site of block. This mechanism may be a more plausible explanation of complete correction of RBBB. The transition from actively bypassing a more proximal RBBB to just passive pre-excitation of $\mathrm{RV}$ free wall at lower voltage may explain the change from complete resolution of RBBB to partial resolution of RBBB.

More than one parallel pathway with different capture thresholds would also explain the voltage dependent phenomenon.

The novel effect of NS-HBP in correcting a distal RBBB may seemingly be at odds with previous reports which suggest that given the longitudinal dissociation of the His bundle $(21,22)$ only pacing the His bundle distal to site of block would correct bundle branch block (1,2). However, it appears that the narrowed QRS they described as 'normal range, not normal activation' (1), 'with stim-Q interval shorter than H-V interval' $(1,2)$, would be similar to the NS-HBP QRS complex we observed with high voltage pacing. It is also likely that our use of 12 lead ECG with filter setting of 0-100 Hz allowed easier visualization of the delta wave as opposed to fewer leads with filter setting of $0-20 \mathrm{~Hz}$ in previous studies (1).

El Sherif et al also reported that in some cases higher pacing voltage was required to narrow the QRS complex and in discussing their results state that longitudinal dissociation in His bundle itself would only explain their conclusions if the transverse interconnections between the longitudinally dissociated fibers $(23,24)$ become functionally inoperative and the conduction block was indeed located in proximal His bundle in all patients (2).

In summary, our previous observation that ventricular activation time decreases with increased pacing voltage in NS-HBP $(5,15)$ (manuscript in review), is extended in this study with the novel finding of greater reduction of RBBB with higher voltage and there appears to be a stark difference between selective and non-selective His bundle pacing on correction of distal block. While we have attempted to explain the mechanisms involved with our current understanding of the $\mathrm{A}-\mathrm{V}$ conduction system, the answers may well lie in the less well understood molecular biology of the His region $(13,14,25)$. Further studies are needed to understand non-selective His bundle pacing, which appears to be distinctly different from the septal paced complex with HVAT of 146 +-26 ms reported by Vassallo et al (26), or the description of the 'wide paced septal complex' to which the term 'non-selective His bundle pacing' was originally applied. (27).

Fig. 6: Myocardial activation models in normal QRS, with non-selective His bundle pacing. 

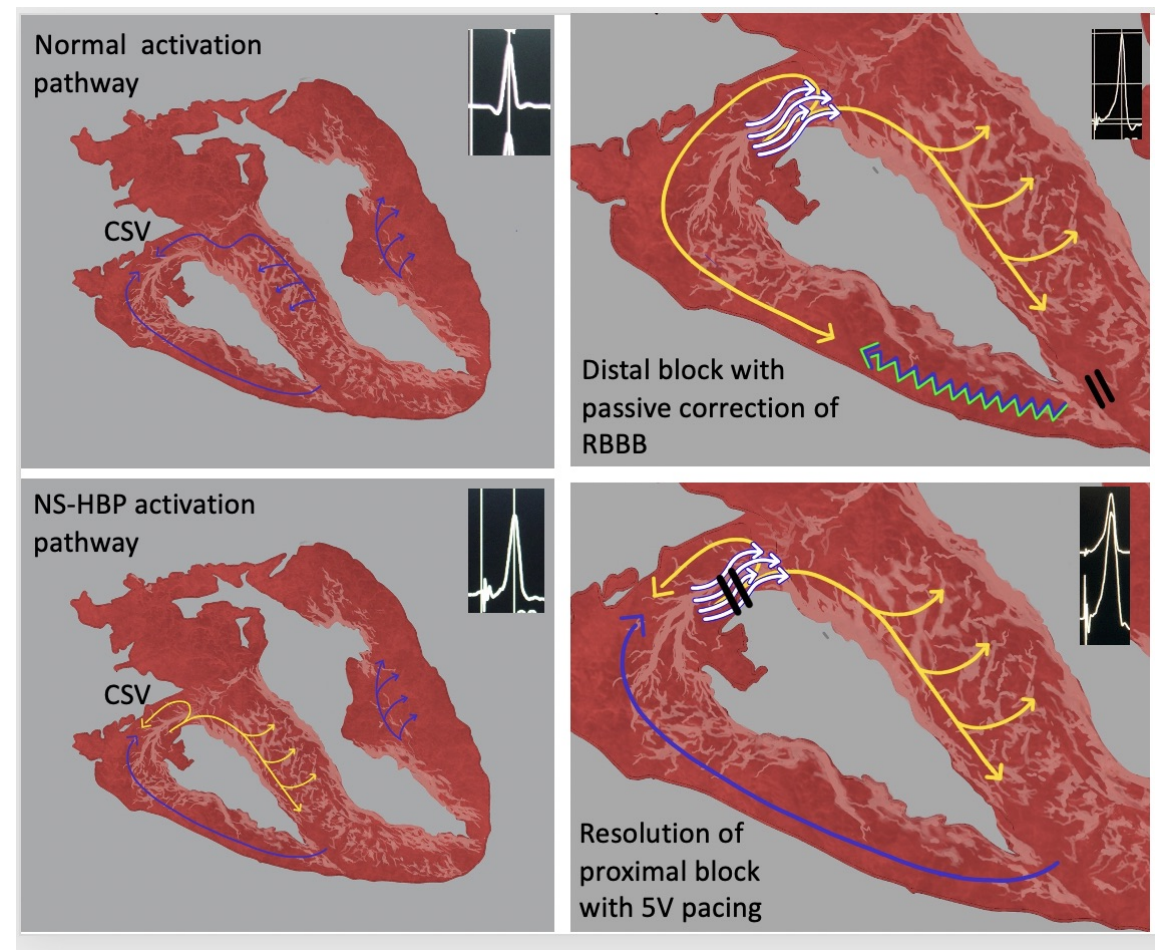

The top left panel shows normal ventricular activation (blue arrow). Note left to right septal activation, then superiorly to septal border of the crista supraventricularis (CSV) and meets the RV free wall wavefront exiting from right bundle branch

The left lower panels show a possible pathway of the of the parallel wavefront (yellow arrow) which, after it reaches the septum moves superiorly to CSV towards the RV free wall. This early rightward activation may result in counterclockwise rotation of the QRS complex.

The right upper panel shows that in RBBB, the parallel wavefront after reaching the CSV and finding an unexcited RV free wall it continues rightward and decreases the duration of S wave. At higher voltage the parallel wavefront may pre-excite larger portion of RV free wall than at lower voltage with slower conduction. The degree of fusion would determine the duration of $\mathrm{S}$ wave while the block remains unresolved.

Right lower panel shows an alternative mechanism where the parallel pathway connects to the His Purkinje system distal to site of block. At higher voltage the parallel wavefront would arrive at this site earlier and no RBBB is seen, and at lower voltage the slower conduction in parallel wavefront allows conduction to occur via His bundle with manifest RBBB.

\section{Limitations}

There are many difficulties in trying to explain phenomenon observed following active fixation of pacing lead with electrophysiologic principles obtained using atraumatic diagnostic catheters. The mechanism will remain a hypothesis until further studies increase our understanding of the complex area surrounding the His bundle.

No high-density mapping data was obtained to determine the exact pathway of the pre-excitation wavefront and we relied mainly on analysis of surface ECG.

Clinical Significance 
Non-selective His bundle pacing may, in essence, be activating an additional pathway with behavior suggestive of a 'parallel His bundle' (25). In patients with His bundle disease, NS-HBP may deserve consideration as preferred mode of His bundle pacing.

Our study also suggests that the paradigm of lowering pacing voltage to prolong battery life may not apply to His bundle pacing as correction of conduction block often occurs at higher voltage pacing.

\section{References}

1. Narula OS. Longitudinal dissociation in the His bundle. Bundle branch block due to asynchronous conduction within the His bundle in man. Circulation. 1977;56(6):996-1006.

2. El-Sherif N, Amay YLF, Schonfield C, et al. Normalization of bundle branch block patterns by distal His bundle pacing. Clinical and experimental evidence of longitudinal dissociation in the pathologic his bundle. Circulation. 1978;57(3):473- 483.

3. Vijayaraman P, Dandamudi G, Zanon F, et al. Permanent His bundle pacing: Recommendations from a Multicenter His Bundle Pacing Collaborative Working Group for standardization of definitions, implant measurements, and follow-up. Heart Rhythm. 2018;15(3):460-468.

4. Sharma PS, Naperkowski A, Bauch TD, et al. Permanent His Bundle Pacing for Cardiac Resynchronization Therapy in Patients with Heart Failure and Right Bundle Branch Block. Circ Arrhythm Electrophysiol. 2018;11(9).

5. Mahmud R, Jamal S, Musheinesh M. Voltage dependent conduction abnormalities in His bundle pacing in patients without His Purkinje system disease. J Electrocardiol. 2019;59:1-6

6. Kupersmith J, Krongrad E, Waldo AL, Conduction Intervals and Conduction Velocity in the Human Cardiac Conduction System. 1973 Circulation;47(4):776- 785

7. Joyner RW. Mechanisms of unidirectional block in cardiac tissues. Biophysical Journal.

8. Boineau JP, Spach MS. The relationship between the electrocardiogram and the electrical activity of the heart. Journal of Electrocardiology. 1968;1(1):117- 124.

9. Dodge FA, Cranefield PF Normal and abnormal conduction in the heart. Nonuniform conduction in cardiac Purkinje fibers. Futura Publishing Company Inc, Mount Kisco New York 1998. 379p.

10. Myerburg RJ. Nilsson K, Befelev B, Castellanos A, Gelband H. Transverse spread and longitudinal dissociation in distal A-V conducting system. J Clin Invest 1977;52: 885-895

11. Roth B.J., "A mathematical model of make and break electrical stimulation of cardiac tissue by a unipolar anode or cathode," in IEEE Transactions on Biomedical Engineering, vol. 42, no. 12, pp. 1174-1184, Dec. 1995, doi: 10.1109/10.476124.

12. Sambelashvili AT, Nikolski VP, and Efimov IR Am J Physiol Heart Circ Physiol 286: H2183-H2194, 2004.

13. Hucker WJ, McCain ML, Laughner JI, Iaizzo PA, Efimov IR. Connexin 43 expression delineates two discrete pathways in the human atrioventricular junction. Anat Rec (Hoboken). 2008;291(2):204-215.

14. George SA, Faye NR, Murillo-Berlioz A, Lee KB, Trachiotis GD, Efimov IR. At the Atrioventricular Crossroads: Dual Pathway Electrophysiology in the Atrioventricular Node and its Underlying Heterogeneities. Arrhythm Electrophysiol Rev. 2017;6(4):179-185.

15. Jamal S, Bailey B, Mahmud R. Comparison of Ventricular Activation Time in Sinus and His Bundle Paced Beats. Circulation Nov 2020 Abstr.

16. Durrer D, van Dam RT, Freud GE, Janse MJ, Meijler FL, Arzbaecher RC. Total excitation of the isolated human heart. Circulation. 1970;41(6):899-912.

17. De Almeida MC, Araujo M, Duque M, Vilhena V. Crista Supraventricularis Purkinje Network and its Relation to Intraseptal Purkinje Network. Anat Rec (Hoboken). 2017;300(10):1793-1801.

18. De Almeida MC, Stephenson RS, Anderson RH, Benvenuti LA, Loukas M, Aiello VD. Human subpulmonary infundibulum has an endocardial network of specialized conducting cardiomyocytes. Heart Rhythm. 2020;17(1):123-130

19. Amer NS, Stuckey JH, Hoffman BF, Cappelletti RR, Domingo RT. Activation of the interventricular septal myocardium studied during cardiopulmonary bypass. Am Heart J. 1960;59:224-237.

20. James TN. Anatomy of the crista supraventricularis: its importance for understanding right ventricular 
function, right ventricular infarction and related conditions. J Am Coll Cardiol. 1985;6(5):1083-1095.

21. Kaufman R, Rothberger CJ: Beitrage zur entstehungsweise extra- sysolischer allorhythmien (Zweite Mitteilung). Z Gesamte Exp Med 7: 199, 1919

22. Sciacca A, Sangiorgi M: Trouble de la conduction intraventriculaire droite duala lesion dutronc commun dufaiseaude His. ActaCardiol 2: 486, 1957

23. Lazzara R, Yeh BK, Samet P: Functional transverse interconnections within the His bundle and the bundle branches. Circ Res 32: 509, 19739.

24. Bailey JC, Spear JF, Moore EN: Functional significance of transverse conducting pathways within the canine bundle of His. Am J Cardiol 34: 790, 1974

25. Personal communication with Dr. Igor Effimov

26. Vassallo, J. A., Cassidy, D. M., Miller, J. M., Buxton, A. E., Marchlinski, F. E., \& Josephson, M. E. (1986). Left ventricular endocardial activation during right ventricular pacing: Effect of underlying heart disease. Journal of the American College of Cardiology,7 (6), 1228-1233. doi:10.1016/s07351097(86)80140-1

27. Williams DO, Scherlag BJ, Hope RR, El-Sherif N, Lazzara R, Samet P. Selective versus non-selective His bundle pacing. Cardiovascular Research1976;10(1):91-100. doi:10.1093/cvr/10.1.91 